\title{
Matter wave compactons in deep optical lattices with strong nonlinearity management
}

\author{
M. Salerno ${ }^{1}$, F. Kh. Abdullaev ${ }^{2}$ \\ ${ }^{1}$ Dipartimento di Fisica "E.R. Caianiello", CNISM and INFN - Gruppo Collegato \\ di Salerno, Università di Salerno, Via Giovanni Paolo II, 84084 Fisciano (SA), Italy \\ ${ }^{2}$ Deparment of Physics, Kulliyyah of Science, International Islamic University Malaysia, \\ 25200 Kuantan, Pahang, Malaysia \\ salerno@sa.infn.it
}

PACS 42.65.-k, 42.81.Dp, 03.75.Lm DOI 10.17586/2220-8054-2015-6-6-742-750

Compacton matter waves are considered in Bose-Einstein condensates (BEC) and in binary BEC mixtures, trapped in deep optical lattices in the presence of strong and rapid periodic time modulations of the atomic scattering length, are considered. For this, we derive vector averaged discrete nonlinear Schrödinger equations (DNLSE) and show that compacton solutions of different types can exist as stable excitations. Stability properties are studied by linear analysis and by direct numerical integrations of the DNLSE system.

Keywords: Bose-Einstein condensates, wave compactons, solitons, nonlinear Schrödinger equation.

Received: 1 November 2015

\section{Introduction}

Periodic management of nonlinear wave system parameters is a very attractive technique for the generation of solitons with new types of properties [1]. Examples of the management technique in continuous systems are the dispersion management of solitons in optical fibers which allows one to improve communication capacities [2], and the nonlinearity management of 2D and 3D Bose-Einstein condensates (BEC) or optically layered media which provides partial stabilization against collapse in the case of attractive interatomic interactions [3]. In discrete systems, the diffraction management technique was used to generate spatially discrete solitons with novel properties $[4,5]$ which have recently been observed in experiments [5]. The suppression of the inter-well tunneling was experimentally observed for light propagation in waveguide arrays [6] and for BEC's in strongly driven optical lattices [7]. In these cases, however, the system is typically subjected either to resonant modulations of the dispersion (coupling between waveguides for the case of light propagation) or to external linear forces (optical lattice shaking in the BEC case). The inhibition of the inter-well tunneling, however, may also become possible in the presence of fast periodic time variations in the nonlinearity, the so called strong nonlinearity management (SNLM). In this case, new phenomena can arise, such as strong localization and formation of (discrete counterparts to) the so called compactons [8] e.g. localized nonlinear waves with compact support.

The aim of the present work is investigate discrete compactons solutions of the discrete nonlinear Schrödinger equation (DNLS) system [9] subjected to SNLM both for single component BEC and for vectorial (binary) BEC mixtures. Contrary to ordinary solitons, the amplitude of a compacton was shown to reduce exactly to zero outside of the localizing domain, implying the total suppression of the inter-well tunneling at the compacton edges. For this, we derive the averaged DNLSE and show that compacton solutions of different 
types can exist as stable excitations [10]. The stability properties are studied by linear analysis and by direct numerical integrations of the DNLSE system. Similar results will also be derived for arrays of binary BEC mixtures described by the vector DNLSE equation in the presence of inter-species scattering length SNLM.

\section{The model}

The dynamical lattice considered here is the well known DNLSE [9]:

$$
i \dot{u}_{n}+\kappa\left(u_{n+1}+u_{n-1}\right)+\left(\gamma_{0}+\gamma(t)\right)\left|u_{n}\right|^{2} u_{n}=0,
$$

which serves as a prototypical model for both matter waves in BEC arrays and for light propagation in arrays of optical waveguides. In the BEC context, $\kappa$ quantifies the coupling (tunneling of matter) between adjacent wells of the optical lattice, $t$ represents the time and $\gamma_{0}$ and $\gamma(t)$ represent the constant and the modulated part of the interatomic interaction (nonlinearity), respectively. In this case the management corresponds to the periodically variable in time the atomic scattering length which can be achieved by the Feshbach resonance technique. In the optical context, the time $t$ should be replaced by the propagation distance $z, \kappa$ quantifies the coupling between adjacent waveguides, and the nonlinear management consists of a periodic spatially variable Kerr nonlinearity around a constant value $\gamma_{0}$. In the following, we shall refer to the BEC context and assume $\gamma(t)$ to be a periodic, $\gamma(t)=\gamma(t+T)$, and rapidly varying function of time of the form: $\gamma(t)=\frac{\gamma_{1}}{\varepsilon} \cos \left(\Omega \frac{t}{\varepsilon}\right)$, with $\varepsilon \ll 1$ and $T=2 \pi / \Omega$ the period.

To investigate the existence of discrete compacton solitons in this model, we shall derive averaged equations over rapid modulations, using the method developed in [11]. Following this approach, we introduce the new variables $v_{n}$ related to the field $u_{n}$ as:

$$
u_{n}(t)=v_{n}(t) e^{i \Gamma(t)\left|v_{n}\right|^{2}(t)}, \Gamma(t)=\frac{1}{\epsilon} \int_{0}^{t} d t \gamma_{1}\left(\frac{t}{\epsilon}\right) .
$$

Substituting this expression into Eq.(1) and averaging the resulting equation over the period of the rapid modulation, we obtain:

$$
\begin{aligned}
i v_{n, t}= & -\alpha \kappa v_{n}\left[\left(v_{n+1} v_{n}^{*}+v_{n+1}^{*} v_{n}\right) J_{1}\left(\alpha \theta_{+}\right)+\left(v_{n-1} v_{n}^{*}+v_{n-1}^{*} v_{n}\right) J_{1}\left(\alpha \theta_{-}\right)\right]- \\
& \kappa\left[v_{n+1} J_{0}\left(\alpha \theta_{+}\right)+v_{n-1} J_{0}\left(\alpha \theta_{-}\right)\right]-\gamma_{0}\left|v_{n}\right|^{2} v_{n},
\end{aligned}
$$

where $J_{n}(x)$ is the Bessel function of order $n, \theta_{ \pm}=\left|v_{n \pm 1}\right|^{2}-\left|v_{n}\right|^{2}$ and $\alpha=\gamma_{1} / K$. This modified DNLSE has essentially nonlinear neighbor-neighbor interactions and can be put in Hamiltonian form $i \dot{v}_{n}=\frac{\delta H}{\delta v_{n}^{*}}$, with the following Hamiltonian:

$$
H_{a v}=-\sum_{n}\left\{\kappa J_{0}\left(\alpha \theta_{+}\right)\left[v_{n+1} v_{n}^{*}+v_{n+1}^{*} v_{n}\right]+\frac{\gamma_{0}}{2}\left|v_{n}\right|^{4}\right\}
$$

For small $\alpha \theta_{+}$the function $J_{0}$ can be expanded in a series giving the same averaged Hamiltonian for the DNLSE obtained in [12] in the limit of weak nonlinearity management.

\section{Compactly-supported localized modes}

In this section, we demonstrate the existence of exact compactons in this averaged system. We remark that compacton solutions were initially reported as a "mathematical curiosity" of somewhat artificial variants of the DNLSE where linear dispersion is absent [13], by analogy to their continuum siblings [8]. The present setting, however, is in some sense unique in that linear dispersion is not, generally speaking, absent. In fact, there is a linear spectrum for the background state in the linearization of even these compact solutions and 

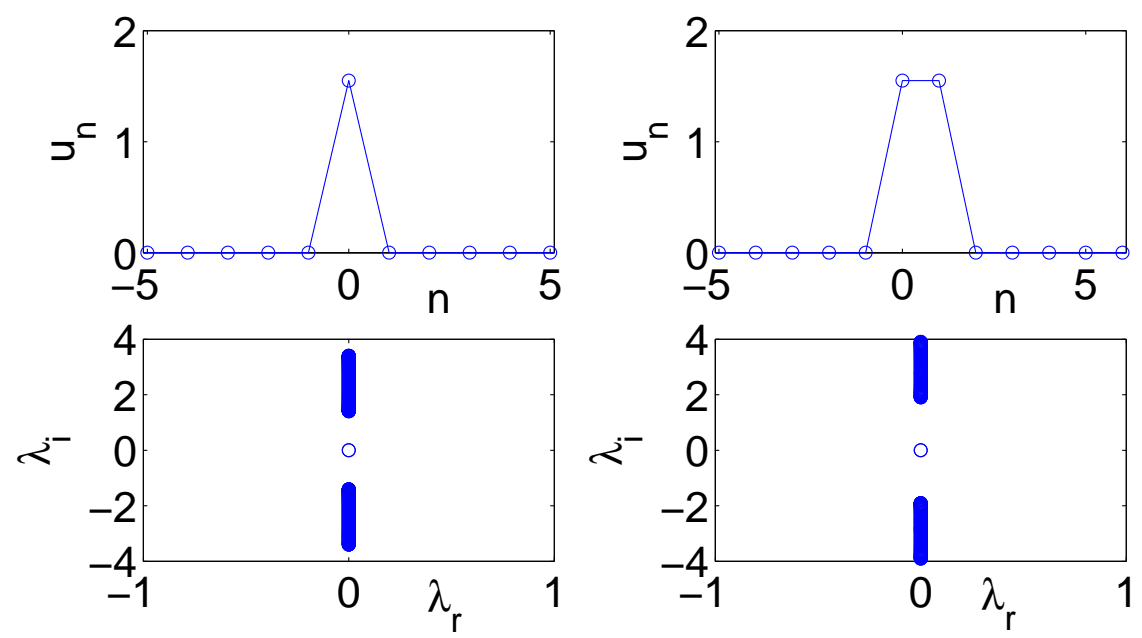

Fig. 1. Typical examples for $\kappa=0.5, \alpha=1$ of compact localized mode solutions of Eq. (4) (top panels) and of the spectral plane $\left(\lambda_{r}, \lambda_{i}\right)$ of their linearization eigenvalues $\lambda=\lambda_{r}+i \lambda_{i}$. 1st column: on-site, 2nd column: intersite in-phase compacton. Remarkably, all solutions are spectrally stable.

can be analytically shown to extend from $[-\mu-2 \kappa,-\mu+2 \kappa]$ and from $[\mu-2 \kappa, \mu+2 \kappa]$. Yet, there exist particular amplitude values which, in essence, completely inhibit the interwell tunneling to the nearest neighbors and hence, enable the formation of such compact structures.

In this regard, we seek stationary solutions of the form $v_{n}=A_{n} e^{-i \mu t}$, for which Eq. (4) becomes:

$$
\begin{array}{r}
\mu A_{n}+\gamma_{0} A_{n}^{3}+\kappa\left(A_{n+1} J_{0}\left(\alpha \theta_{+}\right)+A_{n-1} J_{0}\left(\alpha \theta_{-}\right)\right)+ \\
2 \alpha \kappa A_{n}\left[A_{n+1} A_{n} J_{1}\left(\alpha \theta_{+}\right)+A_{n-1} A_{n} J_{1}\left(\alpha \theta_{-}\right)\right]=0,
\end{array}
$$

and for conditions of tunneling suppression at the last site of vanishing amplitude (edge of the compacton) denoted as $n_{0}$ below. In the setting of Eq. (4), this directly establishes that:

$$
J_{0}\left(\alpha\left|u_{n_{0}+1}\right|^{2}\right)=0 \Rightarrow\left|u_{n_{0}+1}\right|^{2}=2.4048 / \alpha,
$$

which yields the solution based on the first zero of the Bessel function for the "boundary" of the compactly supported site. Then, for $\mu=-\gamma_{0}\left|u_{n_{0}+1}\right|^{2}$, both the condition for compact support at $n_{0} \pm 1$, and the equation for $n=n_{0}$ are satisfied. Hence, Eq. (5) yields a singlesite discrete compacton, which linearization illustrates to be stable (both the solution and its typical linearization are shown in Fig.1).

One can then generalize this type of consideration to two-sites, which are either in phase (2nd column of Fig.1) or out-of-phase (the so-called twisted modes of the 1st column of Fig.2). The only thing that changes here is that in order to satisfy the equation at the non-vanishing sites,

$$
\mu=-\kappa-\gamma_{0}\left|u_{n_{0}+1}\right|^{2} \quad \mu=\kappa-\gamma_{0}\left|u_{n_{0}+1}\right|^{2}
$$



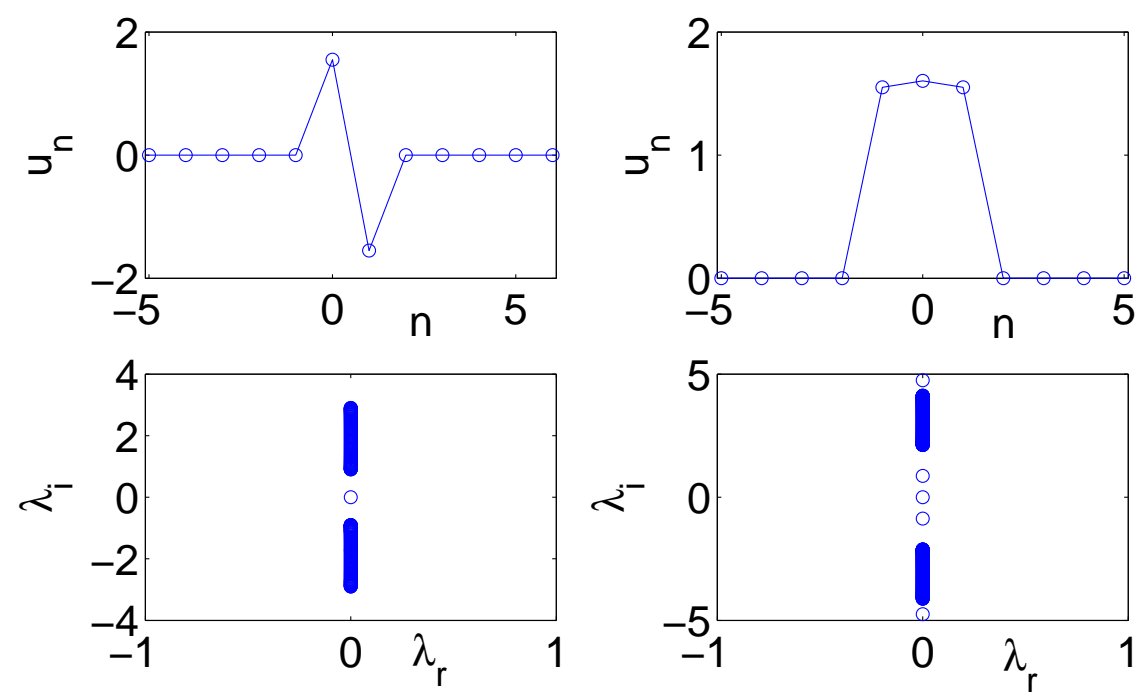

FIG. 2. Compacton solutions of Eq.(4)1st column: inter-site out-of-phase mode, 2nd column: symmetric 3-site compacton.

respectively for the in-phase and out-of-phase two-site modes. We stress that these are exact solutions for the reduced system. Surprisingly, and completely contrary to what is the case for the DNLSE, both of these solutions are spectrally stable, as shown in Fig.2.

Moreover, with some additional effort, one can generalize these considerations to an arbitrary number of sites. As a typical example, a 3 site mode with amplitudes $\left(\ldots, 0, A_{1}, A_{2}\right.$, $\left.A_{1}, 0, \ldots\right)$ will satisfy, in addition to the "no tunneling condition" $J_{0}\left(\alpha A_{1}\right)=0$, the constraints:

$$
\begin{aligned}
& \mu A_{1}+\kappa A_{2} J_{0}\left(\alpha\left(A_{2}^{2}-A_{1}^{2}\right)\right)+ \\
& 2 \alpha \kappa A_{1}^{2} A_{2} J_{1}\left(\alpha\left(A_{2}^{2}-A_{1}^{2}\right)\right)+\gamma_{0} A_{1}^{3}=0, \\
& \mu A_{2}+\kappa A_{1} J_{0}\left(\alpha\left(A_{1}^{2}-A_{2}^{2}\right)\right)+ \\
& 4 \alpha \kappa A_{2}^{2} A_{1} J_{1}\left(\alpha\left(A_{1}^{2}-A_{2}^{2}\right)\right)+\gamma_{0} A_{2}^{3}=0
\end{aligned}
$$

which can be easily solved to yield a solution as the one shown in the 2nd column of Fig.2. Even such more complex solutions which would be highly unstable in the DNLSE are dynamically robust in the present setting. To examine the full nonlinear dynamical stability of these solutions, we considered them as initial conditions both in the averaged Eq. (4), as well as in the full Eq. (1). The results are shown in Fig.3. The single-site results are shown (similar findings were obtained for other modes). The top panel (of large color-bar amplitude) shows the space-time contour map of the solution modulus, while the bottom panel (of small colorbar amplitude) illustrates the deviation from the original solution. To further ensure robustness, a uniformly-distributed random perturbation of small amplitude was added to the original solution. It can clearly be seen that in all cases, both in the averaged equation and in the original system of Eq. (1), the relevant perturbation stays uniformly bounded and never exceeds $2 \%$ of the solution amplitude. The waveforms remain remarkably localized in their compact shape (after a transient stage of shedding off small 


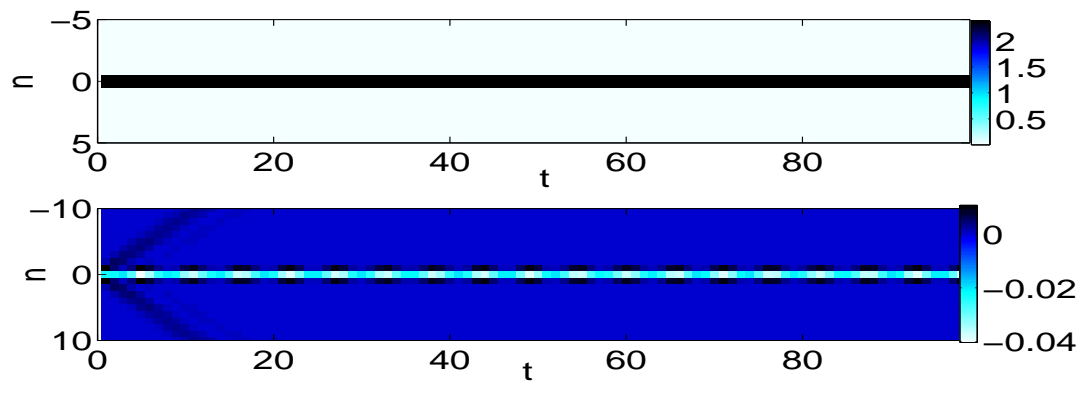

FIG. 3. Time evolution of single-site compacton solution. The top panel shows the evolution obtained from the averaged equation in Eq. (4), while the bottom panel refers to the numerical integration of the original DNLSE system in Eq. (1).

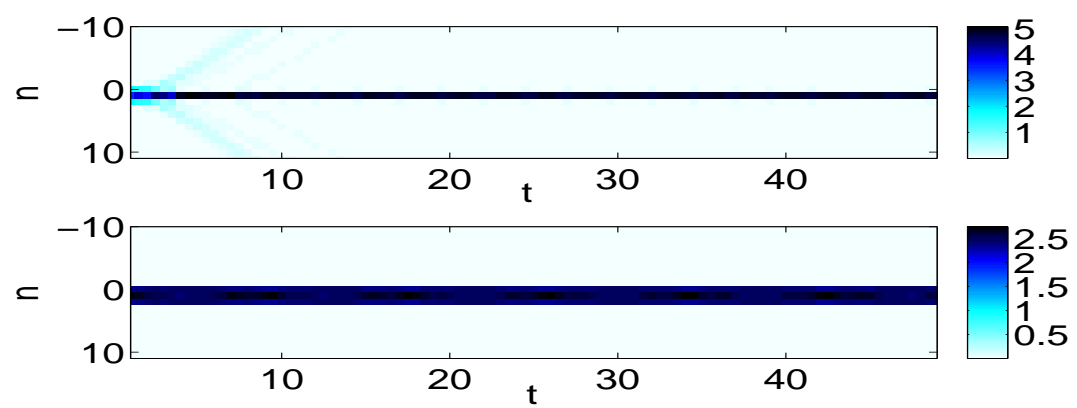

FIG. 4. Time evolution of a perturbed 3-site compacton for $\kappa=1$ decaying into a single-site compacton for $\epsilon=0.1$ (top panel) and remaining stable for $\epsilon=0.025$ (bottom panel). 
amplitude wavepackets). Notice that for Eq. (1), $\gamma(t)=1+\frac{1}{\epsilon} \cos (t / \epsilon)$, with $\epsilon=0.1$ was used.

It should, however, be noted that if one departs from the regime of averaging validity of the averaging, an interesting deviation from the above behavior (and stability) may ensue. An example of this is shown in the panels of Fig. 4. In this case, the 3-site solution was initialized in Eq. (1) with $\epsilon=0.1$ in the top panel, while $\epsilon=0.025$ in the bottom one. While in the latter case, the above-argued robustness of the averaged modes was observed, in the former one, the apparent lack thereof was clearly due to the use of an $\epsilon$ outside of the regime of applicability for averaging approximation. Nevertheless, the resulting evolution confirms the general preference of the system towards settling in compact modes, since despite the large coupling $\kappa=1$ used in this case, the evolution asymptotes to an essentially single-site solution.

\section{Discrete compactons of binary BEC mixtures under SNLM}

The above results can be extended to the case of two-component atomic BEC in a deep OL. In this case, the tight binding approximation leads to the following vector DNLSE [14]:

$$
\begin{gathered}
i \dot{u}_{n}=-\kappa_{1}\left(u_{n+1}+u_{n-1}\right)-\left(\gamma_{1}\left|u_{n}\right|^{2}+\gamma_{12}\left|v_{n}\right|^{2}\right) u_{n}, \\
i \dot{v}_{n}=-\kappa_{2}\left(v_{n+1}+v_{n-1}\right)-\left(\gamma_{12}\left|u_{n}\right|^{2}+\gamma_{2}\left|v_{n}\right|^{2}\right) v_{n},
\end{gathered}
$$

where the overdot stands for time derivative, the coefficients $\kappa_{i}, i=1,2$, are related to the tunneling rates of atoms between neighboring wells of the optical lattice and $\gamma_{12}, \gamma_{i}, i=1,2$ are nonlinear coefficients related to the inter-species $\left(a_{12}\right)$ and intra-species $\left(a_{i i}, i=1,2\right)$ scattering lengths, respectively. Eq. (7) also arises in nonlinear optics where it models the propagation of an electric field in an array of optical waveguides with variable Kerr nonlinearity. In this context, the roles of the nonlinear coefficients $\gamma_{i j}$ correspond to self- and cross-phase modulations of the electric field components, respectively $[15,16]$. Notice that the above two component DNLSE has the Hamiltonian form $\dot{\chi}_{n}=\delta H / \delta \chi_{n}^{*}$ with $\chi_{n}=u_{n}, v_{n}$ and the Hamiltonian $H$ given by:

$$
H=-\sum_{n}\left[\left(\kappa_{1} u_{n+1} u_{n}^{*}+\kappa_{2} v_{n+1} v_{n}^{*}+\text { c.c. }\right)+\frac{1}{2}\left(\gamma_{1}\left|u_{n}\right|^{4}+\gamma_{2}\left|v_{n}\right|^{4}\right)+\gamma_{12}\left|u_{n}\right|^{2}\left|v_{n}\right|^{2}\right] .
$$

Here, the $*$ stands for the complex conjugation and c.c. denotes the complex conjugate of the expression in the parenthesis. Also, notice that the number of atoms $N_{i}=\sum_{n}\left|\chi_{n}\right|^{2}, \quad \chi_{n}=$ $u_{n}, v_{n}$ is conserved for each component. Below, we concentrate on the case of BEC mixtures with fixed (not necessarily equal) intra-species nonlinearities and subjected to strong nonlinear inter-species management of the form:

$$
\gamma_{12}(t) \equiv \gamma_{12}^{(0)}+\gamma_{12}^{(1)}(t)=\gamma_{12}^{(0)}+\frac{\gamma_{12}^{(1)}}{\epsilon} \cos \left(\Omega \frac{t}{\epsilon}\right),
$$

with $\gamma_{12}^{(0)}, \gamma_{12}^{(1)}$ real constants and $\varepsilon$ a small parameter controlling the strength of the modulation as before (strong nonlinearity management corresponding to $\epsilon \ll 1$ with $\Omega, \gamma_{12}^{(1)} \sim O(1)$ ). We remark that although we have considered for simplicity in Eq. (9) a sinusoidal modulation of period $T=2 \pi / \Omega$ in the fast time variable $\tau=t / \epsilon$, results can be extended easily to other types of periodic $\gamma_{12}^{(1)}(t)$ functions. This management setting appears to be quite convenient in experiments since it involves changing only one parameter (for the case of intra-species SNLM see [17]). Moreover, the averaged equations obtained in this case are physically more interesting because nonlinear dispersion terms that involve both components 
could lead, in principle, to novel types of binary compactons. Analogously to what was done for the single component case above, we use the averaging method to eliminate the fast time, $\tau=t / \epsilon$, dependence. In this respect, we perform the following transformation:

$$
u_{n}=U_{n} e^{i \Gamma\left|V_{n}\right|^{2}}, v_{n}=V_{n} e^{i \Gamma\left|U_{n}\right|^{2}},
$$

where $\Gamma$ denotes the antiderivatives of $\gamma_{12}^{(1)(t)}$, e.g. $\Gamma_{12}(\tau)=\frac{\gamma_{12}^{(1)}}{\varepsilon} \int_{0}^{\tau} \cos \left(\Omega \tau^{\prime}\right) d \tau^{\prime}$. By substituting Eq.(10) into Eqs.(7) we obtain:

$$
\begin{aligned}
& i \dot{U}_{n}=i \kappa_{2} \Gamma(\tau) U_{n}\left[V_{n}^{*} X_{1}-V_{n} X_{1}^{*}\right]-\kappa_{1} X_{2}-\left(\gamma_{1}\left|U_{n}\right|^{2}+\gamma_{12}^{0}\left|V_{n}\right|^{2}\right) U_{n} \\
& i \dot{V}_{n}=i \kappa_{1} \Gamma(\tau) V_{n}\left[U_{n}^{*} X_{2}-U_{n} X_{2}^{*}\right]-\kappa_{2} X_{1}-\left(\gamma_{2}\left|V_{n}\right|^{2}+\gamma_{12}^{(0)}\left|U_{n}\right|^{2}\right) V_{n}
\end{aligned}
$$

where $X_{1}=U_{n+1} e^{i \Gamma \theta_{1}^{+}}+U_{n-1} e^{i \Gamma \theta_{1}^{-}}, X_{2}=V_{n+1} e^{i \Gamma \theta_{2}^{+}}+V_{n-1} e^{i \Gamma \theta_{2}^{-}}$and with $\theta_{i}^{ \pm}$denoting the quantities:

$$
\theta_{1}^{ \pm}=\left|U_{n \pm 1}\right|^{2}-\left|U_{n}\right|^{2}, \quad \theta_{2}^{ \pm}=\left|V_{n \pm 1}\right|^{2}-\left|V_{n}\right|^{2} .
$$

The average over the rapid modulation in these equations can easily be done with the help of the relations:

$$
\begin{aligned}
<e^{ \pm i \Gamma(\tau) \theta^{ \pm}}> & =J_{0}\left(\alpha \theta^{ \pm}\right) \\
<\Gamma(\tau) e^{ \pm i \Gamma(\tau) \theta^{ \pm}} & >= \pm i \alpha J_{1}\left(\alpha \theta^{ \pm}\right),
\end{aligned}
$$

where the angular bracket $\langle\cdot\rangle$ denotes the average with respect to the fast time, e.g. $\langle F\rangle \equiv$ $(1 / T) \int_{0}^{T} F d \tau$, while $J_{0}, J_{1}$ are Bessel functions [18] of the first kind for the zero-th and first order respectively, and the parameter $\alpha$ is given by:

$$
\alpha=\gamma_{12}^{(1)} / \Omega \text {. }
$$

The system of averaged equations is then obtained as:

$$
\begin{aligned}
i \dot{U}_{n}= & -\alpha \kappa_{2} U_{n}\left[J_{1}\left(\alpha \theta_{1}^{+}\right)\left(V_{n}^{*} V_{n+1}+V_{n} V_{n+1}^{*}\right)+J_{1}\left(\alpha \theta_{1}^{-}\right)\left(V_{n}^{*} V_{n-1}+V_{n} V_{n-1}^{*}\right)\right] \\
& -\kappa_{1}\left[U_{n+1} J_{0}\left(\alpha \theta_{2}^{+}\right)+U_{n-1} J_{0}\left(\alpha \theta_{2}^{-}\right)\right]-\left[\gamma_{1}\left|U_{n}\right|^{2}+\gamma_{12}^{(0)}\left|V_{n}\right|^{2}\right] U_{n}, \\
i \dot{V}_{n}= & -\alpha \kappa_{1} V_{n}\left[J_{1}\left(\alpha \theta_{2}^{+}\right)\left(U_{n}^{*} U_{n+1}+U_{n} U_{n+1}^{*}\right)+J_{1}\left(\alpha \theta_{2}^{-}\right)\left(U_{n}^{*} U_{n-1}+U_{n} U_{n-1}^{*}\right)\right] \\
& -\kappa_{2}\left[V_{n+1} J_{0}\left(\alpha \theta_{1}^{+}\right)+V_{n-1} J_{0}\left(\alpha \theta_{1}^{-}\right)\right]-\left[\gamma_{2}\left|V_{n}\right|^{2}+\gamma_{12}^{(0)}\left|U_{n}\right|^{2}\right] V_{n} .
\end{aligned}
$$

Here, we only report on some interesting consequences implied by these equations for the simplest case of a bright-bright compacton, which can be searched in the form of for stationary states:

$$
U_{n}=A_{n} e^{-i \mu_{u} t}, V_{n}=B_{n} e^{-i \mu_{v} t}
$$

with $\mu_{u}, \mu_{v}$ chemical potentials of the two atomic species. Substituting these expressions into Eq. $(16,17)$, one gets the following stationary equations:

$$
\begin{array}{r}
\mu_{u} A_{n}+\left(\gamma_{1} A_{n}^{3}+\gamma_{12}^{(0)} B_{n}^{2} A_{n}\right)+\kappa_{1}\left[A_{n+1} J_{0}\left(\alpha \theta_{2}^{+}\right)+\right. \\
\left.A_{n-1} J_{0}\left(\alpha \theta_{2}^{-}\right)\right]+2 \alpha \kappa_{2} A_{n} B_{n}\left[B_{n+1} J_{1}\left(\alpha \theta_{1}^{+}\right)+\right. \\
\left.B_{n-1} J_{1}\left(\alpha \theta_{1}^{-}\right)\right]=0 \\
\mu_{v} B_{n}+\left(\gamma_{2} B_{n}^{3}+\gamma_{12}^{(0)} A_{n}^{2} B_{n}\right)+\kappa_{2}\left[B_{n+1} J_{0}\left(\alpha \theta_{1}^{+}\right)+\right. \\
\left.B_{n-1} J_{0}\left(\alpha \theta_{1}^{-}\right)\right]+2 \alpha \kappa_{1} A_{n} B_{n}\left[A_{n+1} J_{1}\left(\alpha \theta_{2}^{+}\right)+\right. \\
\left.A_{n-1} J_{1}\left(\alpha \theta_{2}^{-}\right)\right]=0
\end{array}
$$


to be solved for the chemical potentials and amplitudes $A_{n}, B_{n}$ of the compacton modes. The compact nature of the solution $\left(A_{i}, B_{i}=0\right.$ outside a finite (small) range of sites), allows one to truncate the above infinite system into a finite number of relations between the above variables, which can be solved exactly.

\subsection{Bright-Bright compactons}

To search for bright-bright (B-B) compacton solutions we need to look for the last sites of vanishing amplitude, i.e. $n_{0} \pm 1$, where the vanishing of the tunneling rate is realized. For a single site B-B compacton, we assume $A_{n_{0}}=a, B_{n_{0}}=b, A_{n_{0} \pm j}=0, B_{n_{0} \pm j}=0$ for all $j \geqslant 1$. Substituting this ansatz in Eqs. $(19,20)$, we obtain the corresponding condition for the compacton existence as:

$$
\begin{aligned}
& J_{0}\left(\alpha a^{2}\right)=0, a^{2}=\xi_{1} / \alpha, \\
& J_{0}\left(\alpha_{2} b^{2}\right)=0, b^{2}=\xi_{2} / \alpha
\end{aligned}
$$

where $\xi_{1}, \xi_{2}$ are zeros (not necessary equal) of the Bessel function $J_{0}$. This condition, together with:

$$
\mu_{u}=-\gamma_{1} a^{2}-\gamma_{12} b^{2}, \mu_{v}=-\gamma_{2} b^{2}-\gamma_{12} a^{2},
$$

gives us the single site B-B compacton pair.

Notice that these equations are the same as for intra-species management case. A big difference with the intra-species management case, however, is the fact that now, the numbers of atoms in the two components of a single site compacton cannot be arbitrary but must be related as follows:

$$
\frac{N_{1}}{N_{2}}=\frac{\xi_{1}}{\xi_{2}}
$$

This relation implies that for a given number of particles in the first $\operatorname{component} N_{1}=a^{2}$ (controlled by the parameter $\alpha$ ), only a discrete set of values for $N_{2}$ are permitted. It is interesting to see if such a state can emerge from generic initial conditions (for example Gaussians). Further research is currently underway to see if this approach is feasible.

\section{Conclusions}

In conclusion, we have shown that in the SNLM limit stable discrete compactons can exist both in one-dimensional BEC trapped in deep optical lattices and in binary BEC mixtures. In this last case, the variety of the compact excitations is larger and includes also dark components in the form of stable bright-dark or dark-dark compactons. The lack of interacting tails in these excitations could be of interest for practical applications. In particular, in the field of nonlinear optics, this could permit one to achieve a maximum rate of information transfer signal in waveguide arrays without disturbing interferences. The solutions considered herein could also lead to experimental observation of discrete compactons both in BEC arrays and in arrays of binary BEC mixtures subjected to SNLM.

\section{Acknowledgements}

MS acknowledge partial support from the Ministero dellIstruzione, dellUniversit e della Ricerca (MIUR) through a PRIN (Programmi di Ricerca Scientifica di Rilevante Interesse Nazionale) 2010 - 2011 initiative (grant \# 2010HXAW77-005). 


\section{References}

[1] B.A. Malomed, Soliton management in periodic systems, Springer-Verlag, Berlin, 2007.

[2] N.J. Smith et al. Enhanced power solitons in optical fibres with periodic dispersion management. Electron. Lett., 1996, 32, P. 54;

I. Gabitov, S.K. Turytsin. Averaged pulse dynamics in a cascaded transmission system with passive dispersion compensation. Optics Lett., 1996, 21, P. 327.

[3] H. Saito and M. Ueda. Dynamically Stabilized Bright Solitons in a Two-Dimensional Bose-Einstein Condensate. Phys. Rev. Lett., 2003, 90, P. 040403;

F.Kh. Abdullaev et al. Adiabatic Dynamics of Periodic Waves in Bose-Einstein Condensates with Time Dependent Atomic Scattering Length. Phys. Rev. Lett., 2003, 90, P. 230402.

[4] M.J. Ablowitz and Z.H. Musslimani. Discrete Diffraction Managed Spatial Solitons. Phys. Rev. Lett., 2001, 87, P. 254102.

[5] I.L. Garanovich, A.A. Sukhorukov, and Yu.S. Kivshar. Nonlinear diffusion and beam self-trapping in diffraction-managed waveguide arrays. Opt. Express, 2007, 15, P. 9547.

[6] A. Szameit, et al. Inhibition of Light Tunneling in Waveguide Arrays. Phys. Rev. Lett., 2009, 102, P. 153901.

[7] H. Lignier et al.. Dynamical Control of Matter-Wave Tunneling in Periodic Potentials. Phys. Rev. Lett., 2007, 99, P. 220403.

[8] P. Rosenau, J.M. Hyman. Compactons: Solitons with finite wavelength. Phys. Rev. Lett., 1993, 70, P. 564;

P. Rosenau. Compact and noncompact dispersive structures. Phys. Lett. A, 2000, 275, P. 193.

[9] P.G. Kevrekidis, The discrete nonlinear Schrödinger equation, Springer-Verlag, Heidelberg, 2009.

[10] F. Kh. Abdullaev, P. G. Kevrekidis, and M. Salerno. Compactons in Nonlinear Schrödinger Lattices with Strong Nonlinearity Management. Phys. Rev. Lett., 2010, 105, P. 113901.

[11] D.E. Pelinovsky et al. Hamiltonian averaging for solitons with nonlinearity management. Phys. Rev. E, 2004, 70, P. 047604.

[12] F.Kh. Abdullaev et al. Intrinsic localized modes in arrays of atomic-molecular Bose-Einstein condensates. Phys. Rev. A, 2003, 67, P. 013605.

[13] P.G. Kevrekidis, V.V. Konotop. Bright compact breathers. Phys. Rev. E , 2002, 65, P. 066614.

[14] U. Shrestha, J. Javanainen, and J. Ruostekoski. Pulsating and Persistent Vector Solitons in a BoseEinstein Condensate in a Lattice upon Phase Separation Instability. Phys.Rev.Lett., 2009, 103, P. 190401.

[15] A. Kobyakov, S. Darmanyan, F. Lederer, E. Schmidt. Dark Spatial Solitons in Discrete Cubic Media with Self- and Cross-Phase-Modulation. Optical and Quantum Electronics, 1998, 30, P. 795.

[16] Gaetano Assanto, Luis A. Cisneros, Antonmaria A. Minzoni, Benjamin D. Skuse, Noel F. Smyth, and Annette L. Worthy. Soliton Steering by Longitudinal Modulation of the Nonlinearity in Waveguide Arrays. Phys.Rev.Lett., 2010, 104, P. 053903.

[17] F. Kh. Abdullaev, M. S.A. Hadi, M. Salerno, and B. Umarov. Compacton matter waves in binary Bose gases under strong nonlinear management. Phys. Rev. A, 2014, 90, P. 063637.

[18] M. Abramowitz and I. A. Stegun, Handbook of Mathematical Functions, National Bureau of Standards, Washington, 1964. 